\title{
Sharing of Data in a Group by Generating Key- Clump
}

\author{
Megha Aigali, Jayashri B. Madalgi and P.B. Patil
}

\begin{abstract}
A great many clients are importing individual information to their companions out in the open system applications in view of capacity of cloud every day. Even company clients are fascinated by storage of cloud because of its advantages, including lower cost, more prominent sharpness and better asset usage. Users are even concerned about leaking of data in the storage of cloud. This data leaks are due to a misbehaving of operator of cloud. To concentrate on users concerns over leaking of data on storage of cloud, an incessant methodology is, information proprietor to encode the information before transferring them to the cloud, later the scrambled information might be recovered and unscrambled by the individual who have the decoding keys. Encrypting data might be tough for data users to find data and afterward specifically regain just the information consists of given catchphrases. Frequent way out for this is, only data owner has to encode conceivable watchwords and transfer to the storage of cloud including encoded data. For recovering information indistinguishable a watchword, information client will send the related aggregate trapdoor towards server of cloud for performing seek operation on the information that is encrypted. Such keys have to be circulated to data users using email, these keys are safely put away and oversaw by the clients in gadgets. Any client may impart a gathering of chose records to a gathering of chose clients. Only data owner has to appropriate a special single total key to information clients for sharing any number of documents, and just client needs to present a single aggregate trapdoor to server of cloud for performing catchphrase seek over any number of shared records.
\end{abstract}

Keywords--- Data Sharing, Encryption, Decryption, Data Privacy.

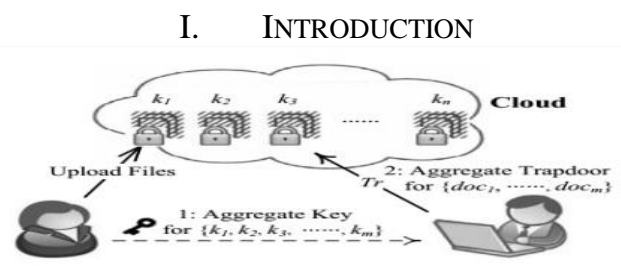

Figure 1: Architecture Diagram of the System

Cloud is a group of servers which gives various types of

Megha Aigali, Department of MCA, GIT, Belgavi, India. E-mail:meghaaigali@yahoo.in

Jayashri B. Madalgi, Assistant Professor, Department of MCA, GIT. Belgavi, India.E-mail: jayashri@git.edu

P.B. Patil, Assistant Professor, Department of MCA, GIT, Belgavi, India. E-mail:pbpatil@git.edu

DOI:10.9756/BIJSESC.8267 internet related services. Cloud computing is used for storing of data and accessing on internet instead of storing data on computer. So that user can access this data from of data is a most important functionality of storage of cloud. We are using public cloud, and storage as a service type to implement this system so that any authorized person can access this system. Using this application, securely, effectively, and flexibly sharing of data with any other group in storage of cloud is possible. In this implementing system there will be generation of public key for encryption, this public key which intern will generate constant size of cipher texts so that efficient assignment of data decryption and downloading authority to any cipher text keyword is possible [9]. One data owner only can generate infinite count of master secret keys, and he can formulate those keys in to compact single unique aggregate key. i.e. Data owner will only generate constant size of aggregated key for any flexible selections of cipher text keywords in storage of cloud, and the data that is encrypted outside of this selected data remain secure. Generated condensed unique aggregate key is expediently shared via a secure channel to data user or this key is stored secretly in smart card which has very limited secure storage [3].

\section{WORK CARRIED}

Sharing of Data in Group by Generating Key-Clump, in which it is possible to share data in storage of cloud confidentially, efficiently, and flexibly.

\section{A. RSA Algorithm}

In this implemented system RSA algorithm is used to encrypt and decrypt files on cloud server [4].

RSA is an algorithm used by modern computers to encrypt and decrypt data. It is an asymmetric cryptographic algorithm. Asymmetric means that there are two different keys. This is also called public key cryptography, because one of them can be given to everyone. The other key must be kept private. It is based on the fact that finding the factors of an integer is hard. RSA stands for Ron Rivest, Adi Shamir and Leonard Adleman, who first publicly described it in 1978. A user of RSA creates and publishes the product of two large prime numbers, along with an auxiliary value, as their public key. The prime factors must be kept secrete. Anyone can use the public key to encrypt a data, but with currently published methods, if the public key is large enough, only someone with knowledge of the prime factors can feasibly decode the data.

RSA involves a public key and private key. The public key can be known to everyone, it is used to encrypt data. Data encrypted using the public key can only be decrypted with the private key. The keys for the RSA algorithm are generated the following way: 
1) Choose two different large random numbers $p$ and $q$.

2) Calculate $n=p q$.

a) $\mathrm{n}$ is the modulus for the public key and the private keys.

3) Calculate the quotient: $\Phi(\mathrm{n})=(\mathrm{p}-1)(\mathrm{q}-1)$.

4) Choose an integer e such that $1<\mathrm{e}<\Phi(\mathrm{n})$, and e is coprime to $\Phi(\mathrm{n})$ i.e., e and $\Phi(\mathrm{n})$ share no factors other than $1 ; \operatorname{gcd}(\mathrm{e}, \Phi(\mathrm{n}))=1$.

a) e is released as the public key exponent.

5) Compute $d$ to satisfy the congruence relation de $=1$ $(\bmod \Phi(\mathrm{n}))$ i.e., $\quad \mathrm{de}=1+\mathrm{k} \Phi(\mathrm{n})$ for some integer $\mathrm{k}$.

a) d is kept as the private key exponent.

The public key is made of the modulus $n$ and the public exponent e. The private key is made of the modulus $\mathrm{n}$ and the private exponent $\mathrm{d}$ which must be kept secret.

\section{B. Generation of Key-Clump}

Aggregate key - Using this aggregate key we can decrypt only shared document of a data owner.

\section{Aggregate key = Master key + Shared files Id's}

Aggregate Trapdoor - aggregate trapdoor is created by converting generated private key to cipher text, which is created to provide search security. i.e., search keyword and shared keys will not be disclosed in cloud.

\section{IMPLEMENTATION}

\section{A. Encrypting Message}

Data owner gives public key ( $\mathrm{n} \&$ e) to data user and keeps private key secrete. Data user wants to send data $\mathrm{M}$ to owner.

First turns $\mathrm{M}$ into a number smaller than $\mathrm{n}$ by using an agreed-upon reversible protocol known as a padding scheme. He then computes the ciphertext c corresponding to:

$$
\mathrm{c}=\mathrm{m}^{\mathrm{c}} \bmod \mathrm{n}
$$

This can be done quickly using the method of exponentiation by squaring. Data user then sends $\mathrm{c}$ to data owner.

\section{B. Decrypting Messages}

Data owner can recover $\mathrm{m}$ from $\mathrm{c}$ by using private key in the following procedure:

$$
\mathrm{m}=\mathrm{c}^{\mathrm{d}} \bmod \mathrm{n}
$$

Given $\mathrm{m}$, data owner can recover the original data $\mathrm{M}$.

The decryption procedure works because first

$c^{d}=\left(m^{e}\right)^{d}=m^{\text {ed }}(\bmod n)$.

Now, since

ed $=1(\bmod \mathrm{p}-1)$ and

ed $=1(\bmod q-1)$

Fermat's little theorem yields

$\mathrm{m}^{\mathrm{ed}}=\mathrm{m}(\bmod \mathrm{p})$ and

$\mathrm{m}^{\mathrm{ed}}=\mathrm{m}(\bmod \mathrm{q})$.

Since $\mathrm{p}$ and $\mathrm{q}$ are distinct prime numbers, applying the Chinese reminder theorem to these two congruences yields

$\mathrm{m}^{\mathrm{ed}}=\mathrm{m}(\bmod \mathrm{pq})$.

Thus,

$c^{d}=m(\bmod n)$.

A working example
Here is an example of RSA encryption and decryption. The parameters used here are artificially small.

1) Choose two random prime numbers

2) $\mathrm{p}=61$ and $\mathrm{q}=53$

3) Compute $\mathrm{n}=\mathrm{pq}$

4) $\mathrm{n}=61 * 53=3233$

5) Compute the quotient $\Phi(\mathrm{n})=(\mathrm{p}-1)(\mathrm{q}-1)$

6) $\Phi(\mathrm{n})=(61-1)(53-1)=3120$

7) Choose e $>1$ coprime to 3120

8) $\mathrm{e}=17$

9) Choose d to satisfy de $=1(\bmod \Phi(\mathrm{n}))$

10) $d=2753$

11) $17 * 2753=46801=1+15 * 3120$.

The public key is $(\mathrm{n}=3233$, e $=17)$.

For a padded message $\mathrm{m}$ the encryption function is: $\mathrm{c}=\mathrm{m}^{\mathrm{e}} \bmod \mathrm{n}=\mathrm{m}^{17} \bmod 3233$.

The private key is $(\mathrm{n}=3233, \mathrm{~d}=2753)$. The decryption function is:

$\mathrm{m}=\mathrm{c}^{\mathrm{d}} \bmod \mathrm{n}=\mathrm{c}^{2753} \bmod 3233$.

For example, to encrypt $\mathrm{m}=123$, we calculate

$\mathrm{c}=123^{17} \bmod 3233=855$

To decrypt $\mathrm{c}=855$, we calculate

$\mathrm{m}=855^{2753} \bmod 3233=123$.

Both of these calculations can be computed efficiently using the square-and-multiply algorithm for modular exponentiation.

\section{RESULT}

\section{A. File Upload}

User is uploading encrypted file.

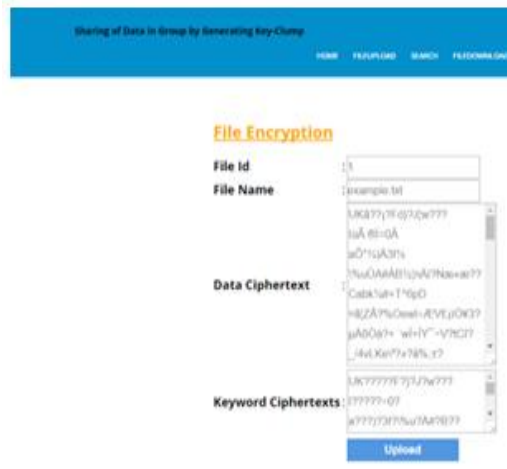

\section{B. Sharing of Data in Group}

Data owner has to select data user to share stored files.
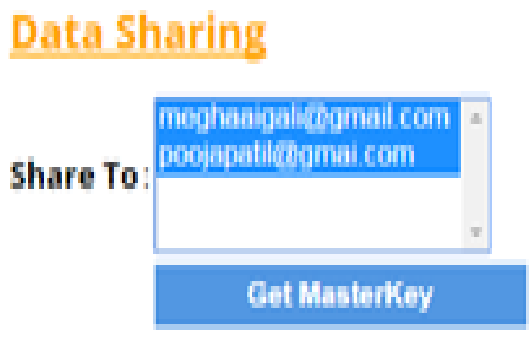


\section{Send Aggregate Key}

Send generated aggregate key to data user.
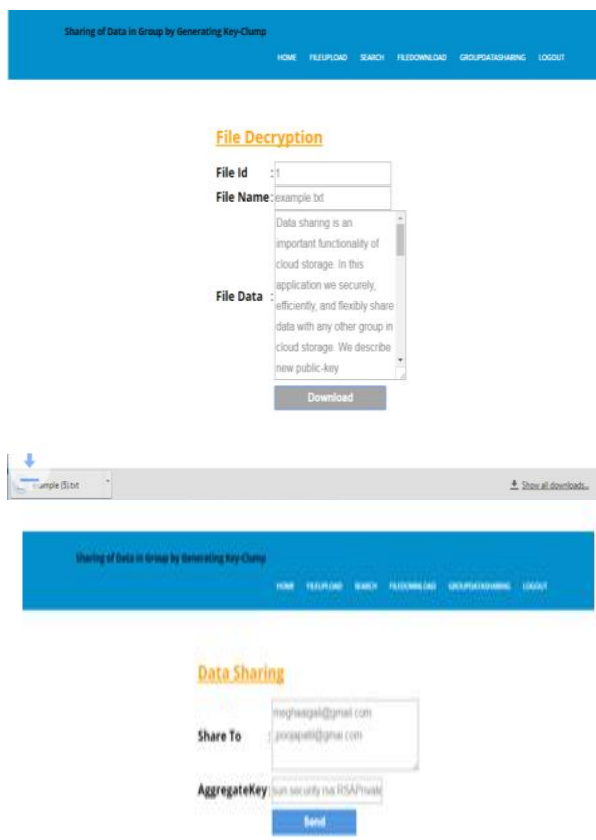

\section{Get Trapdoor}

Enter aggregate key of shared file to generate trapdoor to decrypt file.
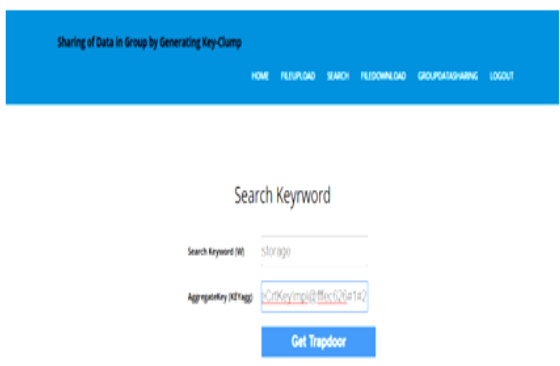

\section{E. Adjust Trapdoor}

Cloud server will adjust trapdoor to perform keyword search operation.
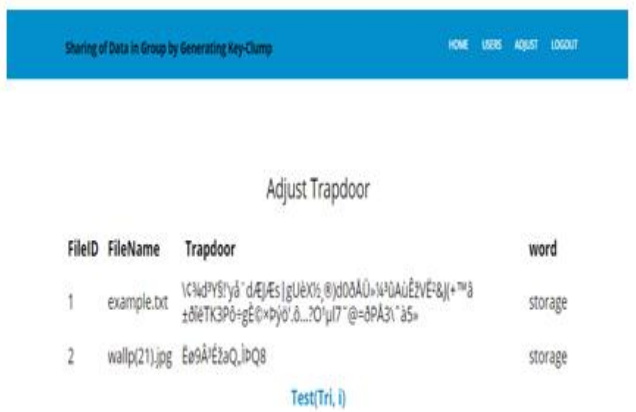

\section{F. File Details}

Shared file detail with data user is displayed on user home page.

\section{G. Decrypt File}

Decrypt shared file using aggregate key.

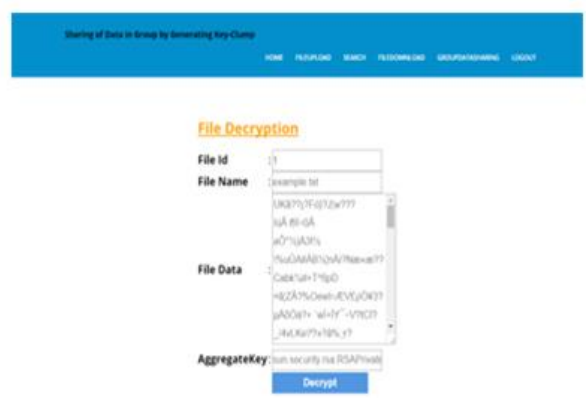

\section{H. Download File}

Downloading shared file in decrypted form.

\section{CONCLUSION}

The difficulties for confidentiality preserving sharing of data system is an open distributed storage in which an information proprietor wants to share out a large number of keys to allow them to access data owner documents. Both investigation and estimation outputs make sure this proposed system can give a successful way of implementing realistic information sharing framework in light of open distributed storage. In proposed system just data owner wants toward send single unique agg-key to information client for distributing large number of records with the information client, and only data client needs to present a solitary unique agg-trapdoor what time user wants to perform search operation on all archives shared by the same information proprietor. Using this trapdoor and search keyword server of cloud will perform search operation and test the result. Then this search result will be sent back to the data user. Using the same agg-key of data owner it is not possible for data user to decrypt or to download the documents that are having similar type of search keyword. That is rest all documents belongs to data owner that are outside the shared documents will be safe.

\section{REFERENCES}

[1] Java fundamentals, A comprehensive Introduction by Herbert Schildt, Dale Skrien, Tata McGrawHill Edition Core Technologies. Second Edition, Vol. 1, 2013.

[2] Marty Hall, Larry Brown, Core Servlet and Java Server Pages.

[3] Java 6 Programming Black Book, Dream tech Press 2012.

[4] W. Stallings, "Cryptography and Network Security - Principles and Practices", $4^{\text {th }}$ Edition, 2009.

[5] R. Curtmola, J. Garay, S. Kamara and R. Ostrovsky. "Searchablesymmetric encryption: improved definitions and efficient constructions", In: Proceedings of the 13th ACM conference on Computer and Communications Security, ACM Press, Pp. 79-88, 2006.

[6] P. Van, S. Sedghi and J.M. Doumen, "Computationally efficientsearchable symmetric encryption", Secure Data Management, Pp. 87-100, 2010.

[7] S. Kamara, C. Papamanthou T. Roeder. "Dynamic searchablesymmetric encryption", Proceedings of the 2012 ACM conferenceon Computer and communications security (CCS), ACM, pp. 965-976, 2012.

[8] D. Boneh, C.G,R. Ostrovsky and G. Persiano, "Public Key Encryption with Keyword Search”, EUROCRYPT 2004, pp. 506C522, 2004. 
[9] Y. Hwang and P. Lee. "Public Key Encryption with Conjunctive Keyword Search and Its Extension to a Multi-user System”, In:PairingBased Cryptography C Pairing, LNCS, Pp. 2-22, 2007.

[10] J. Li, Q. Wang and C. Wang. "Fuzzy keyword search over encrypteddata in cloud computing", IEEE INFOCOM, Pp. 1-5, 2010.

[11] C. Bosch, R. Brinkma and P. Hartel. "Conjunctive wildcard searchover encrypted data", Secure Data Management. LNCS, Pp. 114-127, 2011.

[12] http://java.sun.com

[13] http://www.sourcefordgde.com

[14] http://www.networkcomputing.com/

[15] http://www.roseindia.com 